\title{
Study on PCS7 Heavy Plate Feeder automation control system
}

\author{
Cheng Longquan \\ Sichuan Electromechanical Institute of Vocation and Technology, Panzhihua, Sichuan, China, \\ 617000 \\ E-mail:365799006@qq.com
}

Keywords: PCS7; Heavy Plate Feeder; Automatic Control System; Study

\begin{abstract}
Aiming at problems in the secondary crushing system of a concentrator crushing plant, such as the old-fashioned control mode of heavy plate feeder control system, overload and under-load conditions of the mechanical equipment, large labor intensity of the operators, control lag, and low production efficiency, the research is done on automation design of heavy plate feeder in the secondary crushing system of the crushing plant. The proposed method is to adopt PCS7 system to make automatic detection, control the start-stop and the speed of SIEMENS converter which is used to drive the heavy plate feeder, and use WINCC to design the monitoring picture of the secondary crushing system. The research shows that, with automatic control based on WINCC and PLC, the system becomes flexible, convenient, and stable, and the overload and under-load conditions are eliminated, which further results in lower failure rates. In practical application, through continuous optimization of the improved automatic control system of the heavy plate feeder, better performance on stability, accuracy and failure rates is obtained.
\end{abstract}

\section{Introduction}

In the crushing plant, large pieces of vanadium-titanium magnetite ores from the stope are crushed into standard $12 \mathrm{~mm}$ ores by the feeding system and crushers, with three-stage procedures of "primary crushing--secondary crushing--fine crushing". These crushed ores are then input into the ball mill of the grinding plant. Throughout the whole crushing process, the secondary crushing system plays a vital role, where SIEMENS converter drive 440 is employed in the heavy plate feeder of the ground ore bin to feed the secondary crushing system, sieving system and fine crushing system. The ore feed has an direct affect on the efficiency, energy consumption and system yield. The operator operates the potentiometer on the operation box to adjust the converter operating speed, which further controls the feeding speed(ore quantity).

Several shortcomings exist in the actual production process: Firstly, the operator needs to operate the heavy plate feeder located in the 5 meter deep underground adit, that could leads to high labor intensity. What's worse, the operator is required to use fixed-line to confirm whether the ore input to the next procedure meets requirements, so that the operating speed of the feeder can be adjusted accordingly. To basically fit in with the need of production need, at least six times confirmation and adjustments in one hour are needed, which apparently brings heavy burden on the operator and leads to low productivity. Secondly, equipment under overload or under-load condition increase production costs. For example, facing with the badly damaged drive disk assemblies of overload crushers, a whooping 60 million RMB is spent on maintenance spare parts. In the meantime, 
under-load condition increases the energy consumption of crushers, as 32000 kilowatt-hour more on electricity with total price of 30,000 RMB is spent. Thirdly, the working efficiency is far more satisfactory, failing to feed stably, precisely and in time, equipment such as crushers can only achieve a capacity of about $520 \mathrm{t} / \mathrm{h}$, failing to reach the designed $700 \mathrm{t} / \mathrm{h}$. Low efficiency have a heavy influence on ore yield.

To solve the above-mentioned issues, improvement on the legacy control system of the heavy plate feeder in crushing plant is discussed in this paper.

\section{The Secondary Crushing Process}

Through the belt transmission system, ores in the ground bin are transported to the feeder and then the crusher, where the crushed ores are sieved into the sieving bin. Fig 1 illustrates the whole crushing process.

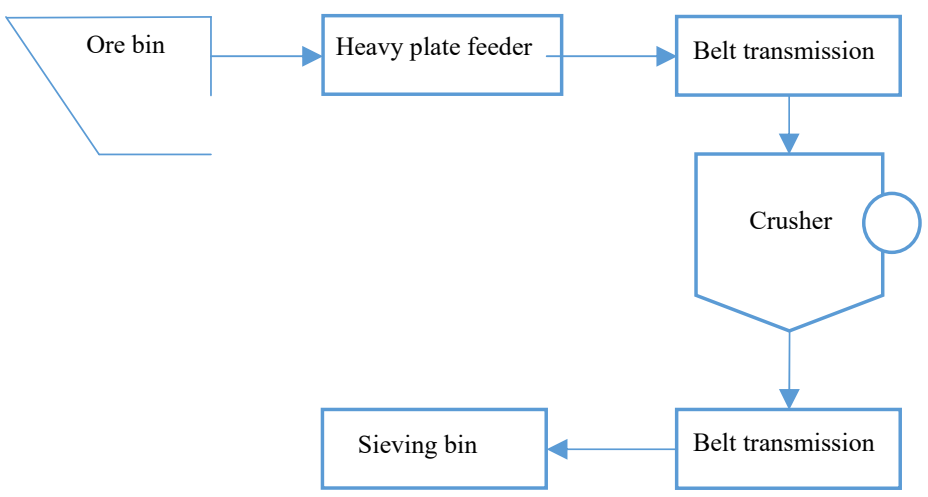

Fig 1. Flow Chart of secondary crushing process

\section{Control System}

Our improved control system adopt closed-loop cascade controlling architecture, including feeding control loop and load control loop. The control principle is shown in Fig 2.

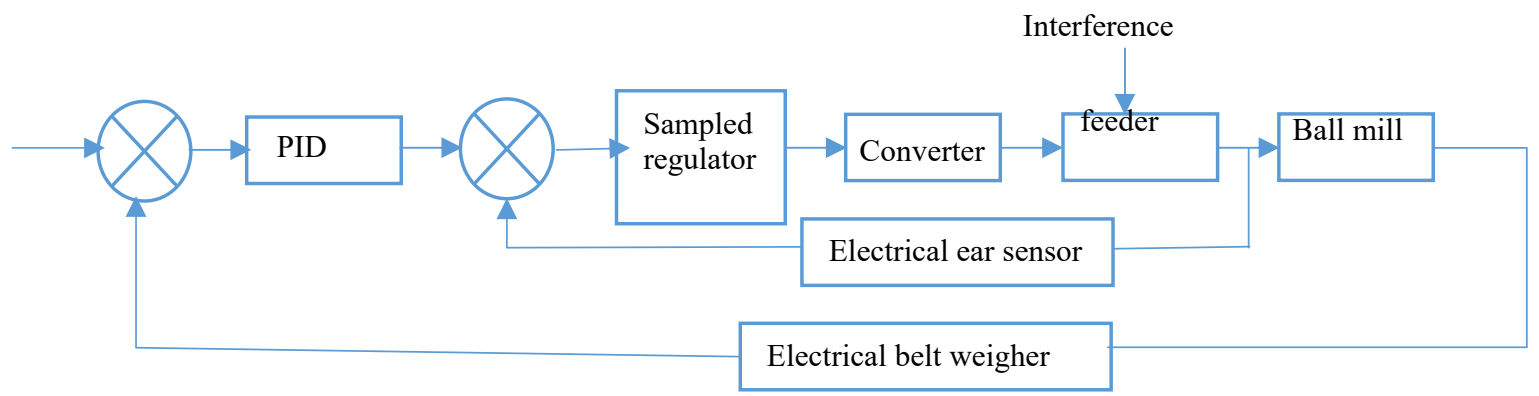

Fig 2. Feeding control system schematic diagram

The inner loop archives constant feed control, while the cascade part archives load control. An electronic belt weigher detects the ore feed, under the measurement and control of feeding control loop, the feeder rotation speed is governed by the converter in order to adjust the ore feed quantity. Said process ensures that the feed quantity be constantly adjusted when the feed set-point keeps the same. During the control, the control period T is equal or greater than the latency $t$. In addition, the PI algorithm is used for floating purpose. Integral separation is applied to optimize the PI algorithm, to decrease the overshoot and shorten adjustment time of the control system. When the error is minimized to a certain range, said integral item can only be effective ${ }^{[1]}$. 
The model algorithm is shown as follows:

$$
\begin{aligned}
u_{i} & =K_{p}\left(e_{i}+K_{i} \cdot \frac{T}{T_{i}} \sum_{j=0}^{i} e_{j} \frac{T_{d}}{T}\right) \\
K_{i} & =1\left(e_{j} \leq \varepsilon\right) \\
K_{i} & =0\left(e_{j}>\varepsilon\right)
\end{aligned}
$$

$K_{P}$ is the proportion coefficient, $I$ is the critical deviation, $T_{i}$ is the integral time, $K$ is the separation coefficient, $e_{i} 、 e_{j}$ is the deviation between the ore feed and the actual ore quantity ${ }^{[2]}$.

Based on the SIEMENS converter drive 440 built-in serial communication interface(SCI), PROFIBUS cable is used to connect the $9^{\text {th }}$ slave station ET200M of the original PROFIBUS-DP field bus serial distributed system to the converter, to make the MM440 converter the $10^{\text {th }}$ slave station $^{[3]}$.

When WINNC has built connection with S7-400PLC through the Ethernet, and S7-400PLC has built connection with 440 converter through PROFBUS-DP, WINNC is able to monitor the variables in S7-400PLC programs. And then S7-400PLC can supervise the PZD zone of the MM440 converter, to achieve remote control on the start-stop and speed giving of the converter ${ }^{[4]}$. The system structure is shown in Fig 3.

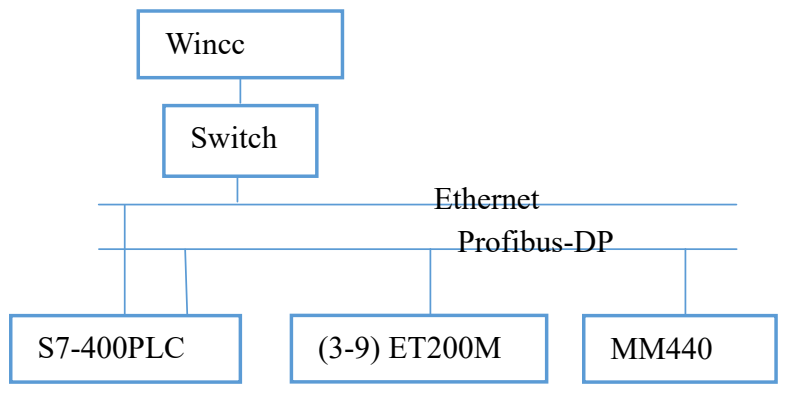

Fig 3. System structure

\section{Hook between MM4400 Converter and PROFIBUS-DP, Parameter Configuration}

\subsection{Converter Installation}

SIMENS converter 440 is chosen, who has superiority in overload ability under low-speed, high-torque output load condition and good dynamic performance ${ }^{[5]}$. Insert the DP communication model matching with the MM440 converter into the SCI. One end of the SIMENS DP bus cable (6XV1830-0EH10) is connected with the DP bus connector of the $9^{\text {th }}$ slave station ET200M, and the terminal resistance is switched from "On" to "Off", meanwhile, the other cable end is connected to the DP communication model to communicate with the converter through its $\mathrm{SCI}^{[6]}$.

\subsection{Hardware Configuration, Hook between Converter and DP network}

Hardware configuration is established on a upper computer installing SIMENS Step7 programming software ${ }^{[7]}$, as shown in Fig4. Hook between Converter and DP network is illustrated in Fig 5. Save and compile the hardware configuration and download it to S7-400PC. 


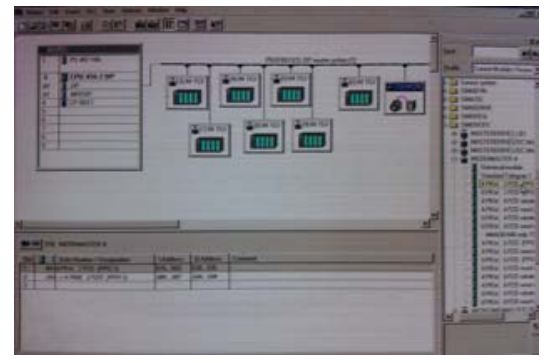

Fig 4 Hardware Configuration

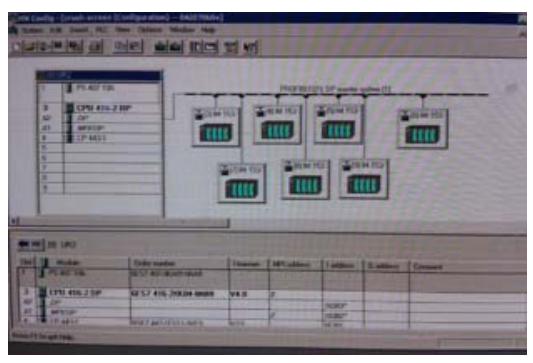

Fig 5.Hook between Converter and DP network

\subsection{Parameter Configuration of converter 440}

To achieve DP bus communication, the parameter of converter 440 should be configured:

P0003: Access configuration: before the DP communication parameter is set, the P0003 parameter is set to 3-expert level, and then it's permitted to configure the DP network parameters, when it's finish, the P0003 parameter is modified to 1-standard level.

P0700 Select digital command signal source: the communication board(CB) is set to 6-com link, to make communication available.

P0719 Command, frequency set-point Configuration: the parameter is set to zero, the command controller switch is used to select converter control command source, and switch between programmable BICO parameters and fixed command/set-point, between command source and set-point signal source.

P0918 CB Address Configuration: Assign the converter's bus address on PROFIBUS-DP to CB. Two possible ways can be applied to set the bus address, one way is to set through the DIP switch on the PROFIBUS template, another is that user input the address. If PROFIBUS template is applied, DIP=0, the assigned address of P0918 is 10. The DIP switch is set to zero when released, so the converter bus address is configured by P0918 during debug period. When DIP is not set to zero, switch setting is preferred, and P0918 is responsible for displaying the set-point of DIP switch.

P1000 selection of frequency set-point: the parameter is set to CB of 6-COM link, the frequency set-point communicates with the CB.

\section{Field Control System}

Given the actual field control situation, we apply PROFIBUS as the bus control format and establish the connection and monitoring between field devices and workshops through distributed digital controlling, so as to accomplish automation between heavy plate feeder control system and field devices.

Functions like automatically controlling the ball mill feeding, and real time monitoring of the process flow, reporting, statistics, remote browsing are involved in the designed system ${ }^{[8]}$.

The CP5611 communication board is installed on both two control machines, the CP342-5 communication processor model is used on PLC, and information interaction is done with PROFIBUS. The control machine displays running state of the monitoring devices in real time, and monitors real-time data such as feeding quantity, operating speed, load curves and bearing temperature. In addition, common functions like inquiry of historical data, setting parameters, generating statistical analysis reports are also involved. PLC program handles the whole control process, corresponding commands are transmitted to individual sites through DP networks, to realize automatic control. In order to realize timely regulation against basic-level production and get a real-time understanding of the production status and device running state, one extra server can be used to network with the scheduling supervisor through the switch device, to meet the demands of 
automatic monitoring and managing. The network topology is shown in Fig $6^{[9]}$.

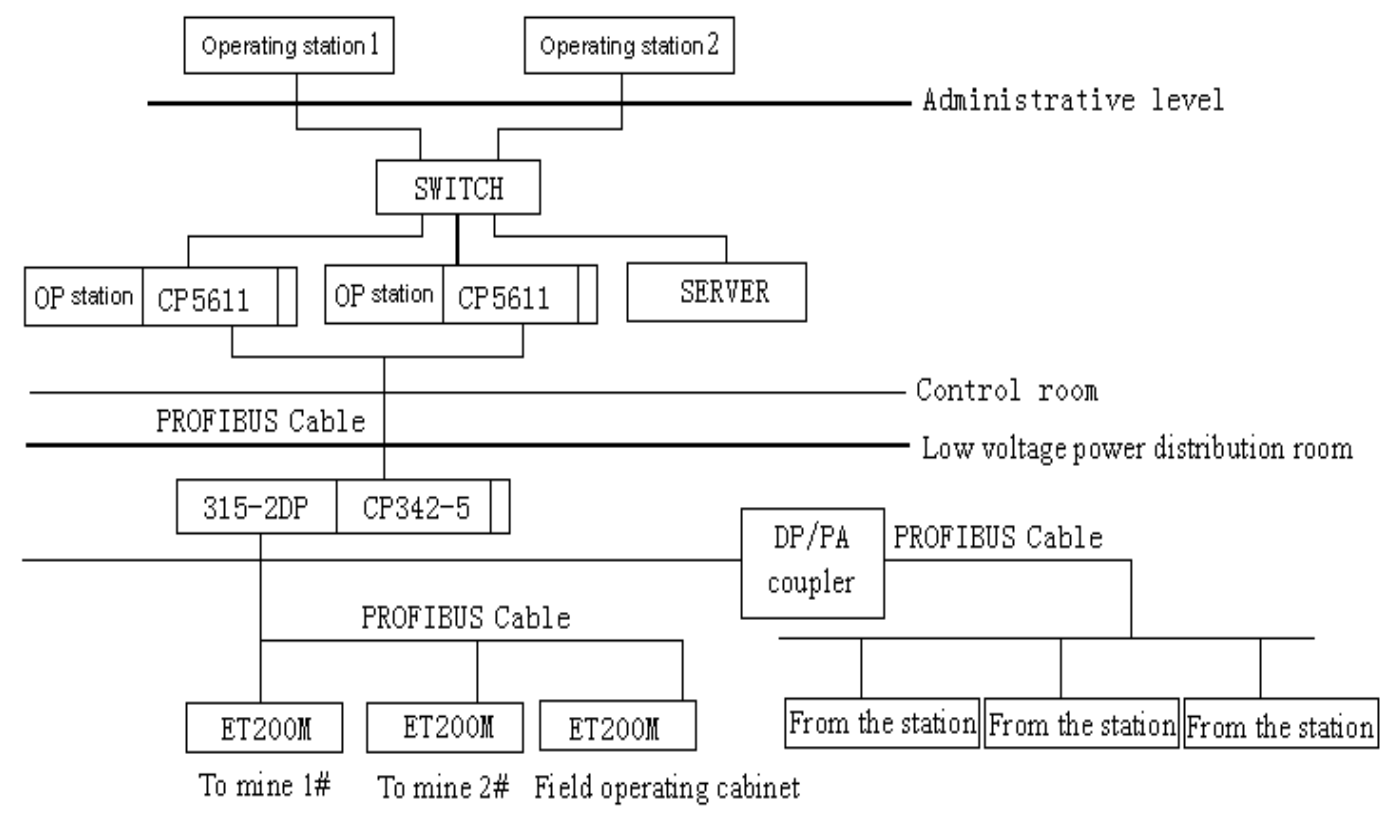

Fig 6 Network topology

\section{Programming design}

\subsection{PLC control program}

The main control program of PLC is made by block calls, main program of PLC is shown in Fig 7 and the control program block is shown in Fig 8.

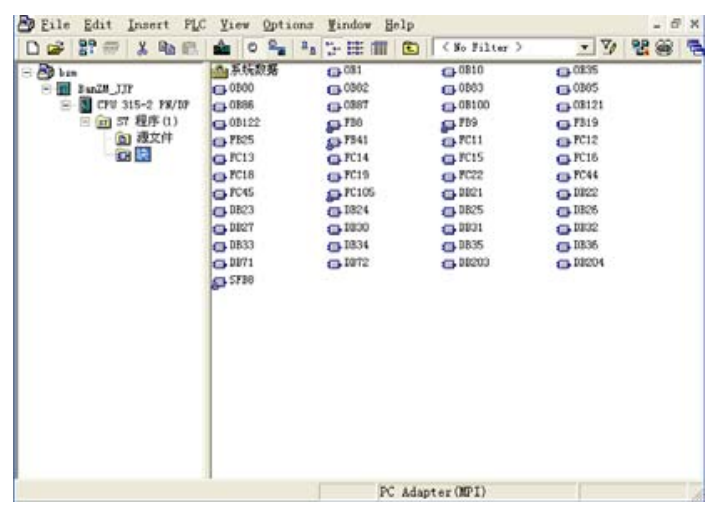

Fig 7. Main program

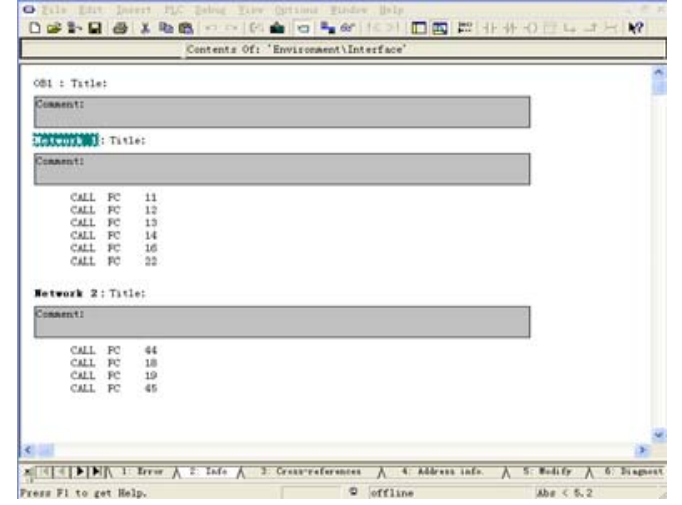

Fig 8. Control program block

\subsection{Communication Program Design between converter and PLC}

PP0 is responsible for connection between the converter and PLC master station, on one hand, master station uses PP0 to transmits the control word (STW), frequency set value to converter 440, on the other hand, converter 440 replies status word (ZSW) nd the actual frequency output (HIW) to PLC master station through PP0 ${ }^{[10]}$. The text structure of PP0 is shown in Fig 9. 


\begin{tabular}{|c|c|c|c|c|}
\cline { 2 - 5 } \multicolumn{1}{c|}{} & PZD1 & PZD2 & PZD3 & PZD4 \\
\hline Master站 $\rightarrow$ MicroMaster440 & STW & HSW & HSW2 & STW2 \\
\hline MicroMaster440 $\rightarrow$ Master站 & ZSW & HIW & ZSW2 & HIW2 \\
\hline
\end{tabular}

Fig 9. PP0 text structure

PZD1-PZD4: process data area, used for converter control and detection, taking 8 bytes.

STW: control word, taking 1 or 2 bytes, used for controlling start-stop, alarm reset of the converter.

HSW: frequency set value, taking 3 or 4 bytes, used for modifying the frequency set value, converter 440 feed HSW back to the PLC master station.

ZSW: status word, taking 1 or 2 bytes, defined by parameter r0052, used for displaying the converter status (running, breakdown).

HIW: actual frequency output, taking 3 or 4 bytes, displaying converter feedback frequency.

\subsection{Program Design of Converter Start, Stop and Running}

Left-click the "Start" button in WINCC, and then M100.0 builds connection with W\#16\#47F converter, the start-up control word is then transmitted via PQW536 to the converter's PZD control area, converter starts. Drag the slider to input certain speed value into MW101, in order to set the speed of the converter via PQM538, meanwhile, the converter transmits the actual speed to MW110 via PIW586. M100.2 coil is "energized", and its auxiliary point M100.2 self complements, right-click the "start" button, M100.0 will be disconnected ${ }^{[11]}$, the start-up program design is shown in Fig 10.

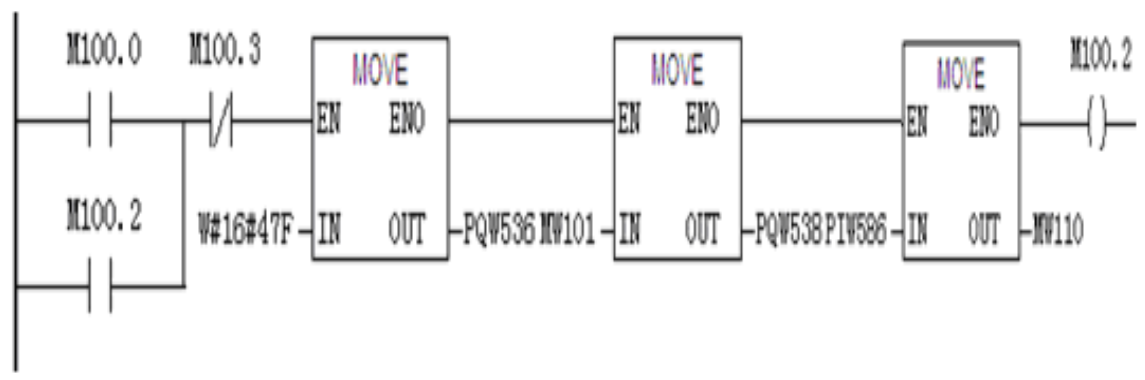

Fig 10. Program design of converter start-up

Left-click the "Stop" button in WINCC, and then M100.1 is connected, if stop control word "W\#16\#47E" is transmitted to PZD control area via PQW536, the converter is stopped. Whereafter, right-click the "Stop" button and M100.1 will be disconnected. The corresponding program design is shown in Fig 11.

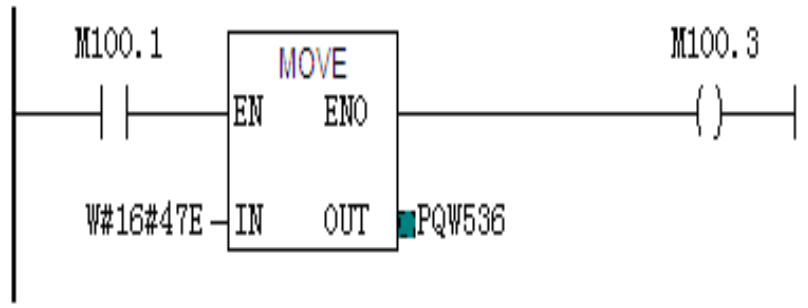

Fig 11. Program design of stopping converter 
The actual speed value of converter is converted from integer data type to long integer type in MW110 and transmitted to MD120, where the long integer data is further converted to float type and transmitted to MD130. The float type data in MD130 is then divided by $1.638400 \mathrm{e}+004$ and transmitted to MD140, where it's multiplied by $5.000000 \mathrm{e}+001$ and then transmitted to MD150. Data output from MD150 is displayed on the input/output domain of WINCC's operating and monitoring screen. The compiled PLC programs are then saved and downloaded into S7-400PLC ${ }^{[12]}$. The converter's operation program is shown in Fig 12.

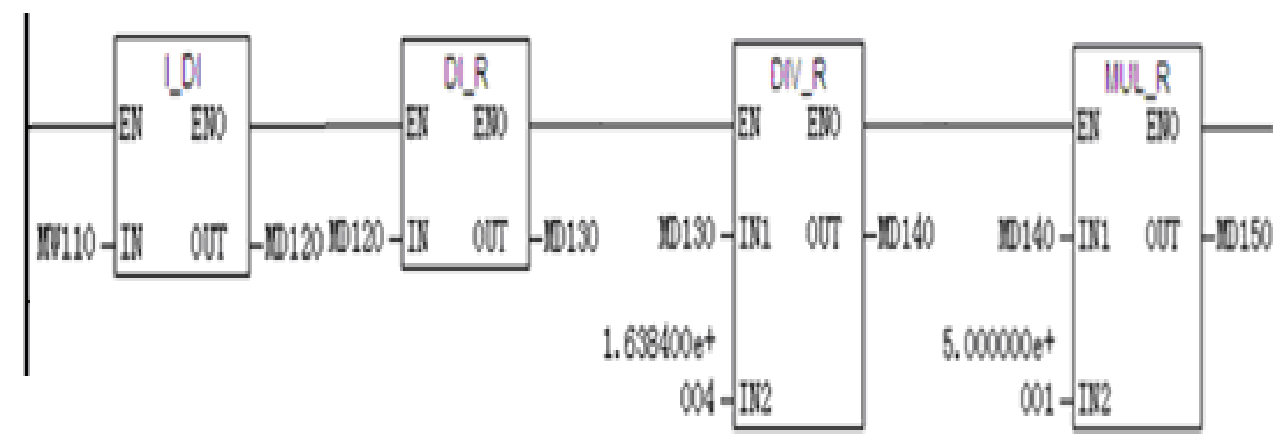

Fig 12. Operation program design

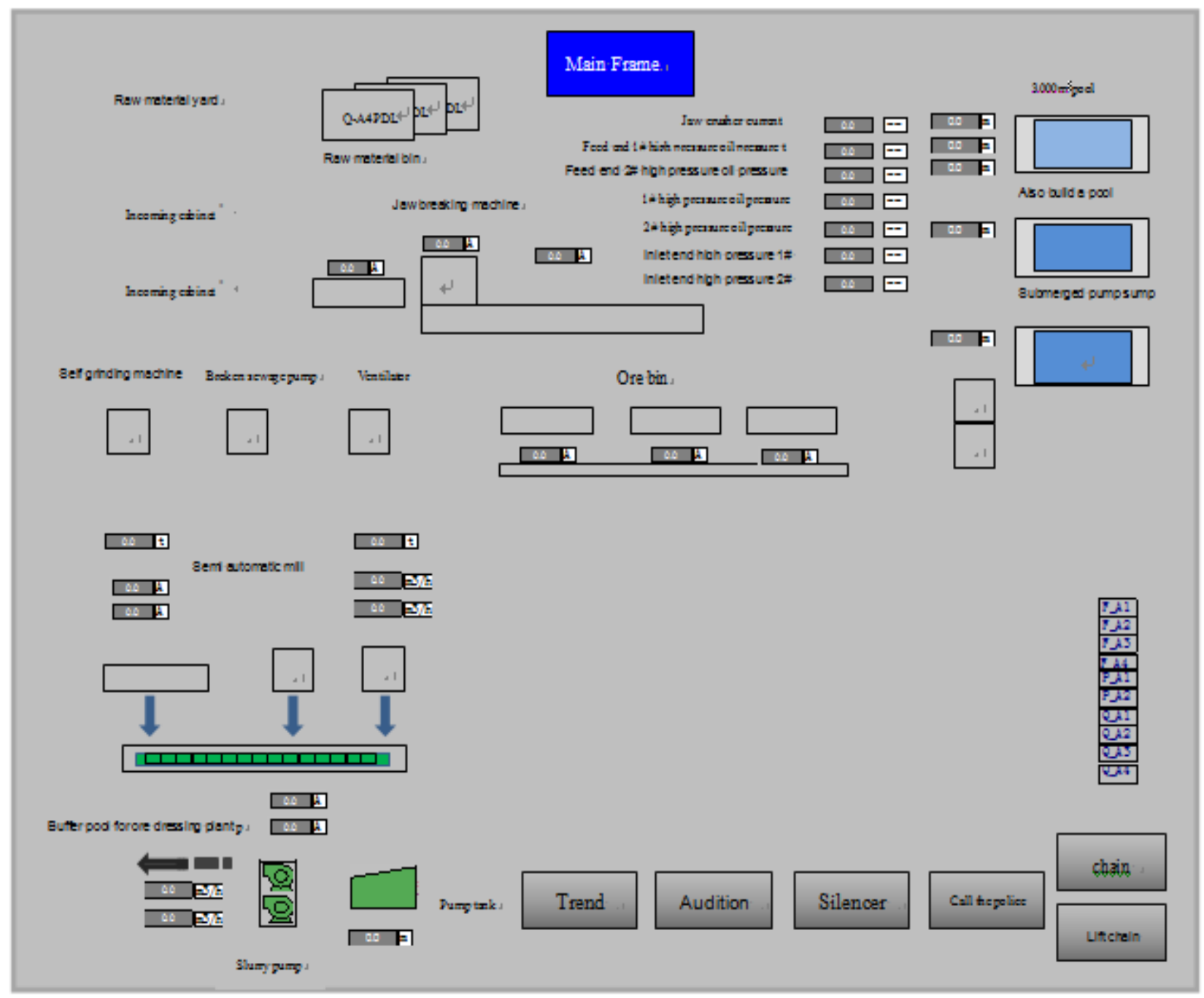

Fig 13.WINCC control interface 


\section{UI design of WINCC Monitoring}

Configure the external variable and processing screen, and the whole screen of bai middle crush_2.PDL is built and saved. When running, click the heavy plate feeder figure on the main screen of secondary crushing system monitoring, and a extra small control screen of the heavy plate feeder will pop up, where we can manipulate the "start" or "stop" button to control the converter to start or stop, drag the slider to change the converter speed, and display the converter in the input/output domain. The WINCC control interface is shown in Fig 13.

\section{Configure the upper computer}

The real-time monitoring interface can be developed in a Windows environment, and WINCC is adopted to develop the upper computer's control UI. The control UI is mainly composed of the following parts:(1) Main interface region, visually and dynamically demonstrating the parameter variation of field devices, simulating the real-time process of ore feeding. The control data comes from the field data and the corresponding variable defined in the kingview software data base. Graphic object is applied to build animation connection between the field data and the variable. (2)Signal interface analysis region: displaying specified signal data and relative tendacy charts, so that operators can analyze the running states, judge the causes and location of operation failure, and make corresponding prediction. (3) Alarm message region: when events that meets the preset alarm condition happens, the corresponding alarm pop-up window and sound alert is triggered until the operator has confirmed to clear the alram. The alarm window is equipped with historical alarm recording system, the events' corresponding process logs are recorded for query and print purpose. (4)Report generation region: in order to handle all production equipment and have a pooled analyses of the production data, the embedded report generating system of WINCC is adopted to generate all kinds of analysis reports and the system function is called to pint these reports.

\section{Conclusions}

The improved automatic control system of heavy plate feeder has a higher automation degree and it's easier to operate. The site operators only need to view the secondary system processing screen and the main screen display to grasp the ore quantity of each stage and the running status of devices. Real time monitoring makes it possible to remote control the speed of heavy plate feeder, which further changes the feeding quantity, so that the whole crushing system is able to operate in a stable and efficient way. The result of field operation demonstrates that the control system is stable and reliable, nearly no breakdown happens during the trial run,. Besides, the system gets very precise ore feeding and the operators' labor intensity decreases greatly. There is no need to adjust the ore feeding manually, routine inspection once an hour is sufficient. Also, with our automatic control system, the ore throughput has reached $700 \mathrm{t} / \mathrm{h}$, and the economic benefit has got on a new stage. The overload and under-load problems of mechanic devices (such as crushers) are solved too, mechanic maintenance caused by overload and energy consumption caused by under-load are nearly zero. In addition, thanks to real-time monitoring, the idle running time is reduced to $4-5$ minutes, production cost is has decreased dramatically.

\section{References}

[1] WU Xing-chun, ZHAO Jing-yan. Application of intelligent PID algorithm in finance temperature control system [J]. Journal of Mechanic \& Electrical Engineering, 2011, (28) 8: 948-959.

[2] ZHANG Yong-ping. Application of integral separating PID control in the system of batching system [J]. China 
hi-tech Enterprises, 2010, 39 (1) : 20-21.

[3] LAN Li, LI Hong-xing. FCS integration based on Profibus-DP [J]. Microcomputer Information, 2007 (23) 6:14-16.

[4] LIU Lin. Design of "transparent" pump station SCADA system based on EtherCAT [J]. Journal of Mechanical \& Electrical Engineering, 2013, 30 (10) : 1289-1290.

[5] YAN Wen-jun, MAO Xue-zhen. Power staion automatic remote monitor system design based on PLC and Wincc[J]. Journal of Mechanical \& Electrical Engineering, 2007, 24 (11) : 43-46.

[6] WANG Guang-fu. Study on a new intelligent PLC with switch management[J] Journal of Industrial and Systems Engineering, 2015, 21 (2): $227-241$.

[7] LIAO Chang-chu. S7-300/400PLC application [M]. Beijing: China Machine Press, 2007.

[8] SUN Jing-min, LI Shi-hou. Design and research on automatic control system of dressing mill [J]. Nonferrous metals (dressing part).2007: 30-41.

[9] XUE Ying-cheng.,HE Jian-qiang. Principles and Application of IPC and configuration control technology [M]. Beijing:China Electrical Power Press,2007.

[10] Wang Guang-fu. Study on PLC with switch management in intelligent manufacturing network[J]. International Journal on smart Sensing and Intelligent Systems, 7 (2), 2014.

[11] Frank D.Petruzella. Programmable logic controllers[M]. 3th Posts \& Telecom Press, 2007.

[12] PAN H Z. Experimental validation of a nonlinear backstep-ping liquid controller for a state coupled two tank system[J]. Control Engineering Practice, 2005(13) : 27-40. 